\title{
Forward $Z$-boson production and the unintegrated sea quark density
}

\author{
F. Hautmann ${ }^{\text {a }}$, M. Hentschinski ${ }^{b, *}$, H. Jung ${ }^{c, d}$ \\ a Theoretical Physics, University of Oxford, Oxford OX1 3NP, United Kingdom \\ b Instituto de Física Teórica UAM/CSIC, Universidad Autónoma de Madrid, E-28049 Madrid, Spain \\ ${ }^{\mathrm{c}}$ Deutsches Elektronen Synchrotron, D-22603 Hamburg, Germany \\ d CERN, Physics Department, CH-1211 Geneva 23, Switzerland
}

Received 9 May 2012; accepted 20 July 2012

Available online 27 July 2012

\begin{abstract}
Drell-Yan production in the forward region at the Large Hadron Collider is sensitive to multiple radiation of QCD partons not collinearly ordered, emitted over large rapidity intervals. We propose a method to take account of these radiative contributions via a factorization formula which depends on the unintegrated, or transverse momentum dependent, splitting function associated with the evolution of the initial-state sea quark distribution. We analyze this formula numerically, and point out kinematic effects from the initialstate transverse momentum on the vector boson spectrum.
\end{abstract}

(C) 2012 Elsevier B.V. All rights reserved.

\section{Introduction}

Many aspects of the experimental program at the Large Hadron Collider (LHC) depend on the analysis of processes at large momentum transfers containing multiple hard scales. As such, they will be influenced by improved formulations of factorization in QCD at unintegrated level [1], which serve in multi-scale processes to control perturbative large logarithms to higher orders of perturbation theory and to describe appropriately nonperturbative physics in the initial and final states of the collision. These formulations involve transverse-momentum dependent (TMD), or unintegrated, parton decay and parton density functions (pdfs) [2].

\footnotetext{
* Corresponding author.

E-mail address: martin.hentschinski@uam.es (M. Hentschinski).
} 
A broad class of such multiple-scale events is given by small- $x$ processes. These processes are one of the main sources of final states in the central region at the LHC [3]. Besides, they are responsible for the sizeable rates of forward large- $\mathrm{p}_{\perp}$ jet production at the LHC, giving rise to new phenomenology in the LHC forward region compared to previous collider experiments, e.g. forward jet physics [4,5]. Small- $x$ TMD factorization and pdfs (see for instance recent reviews in [6,7]) serve for the perturbative resummation of high-energy logarithmic corrections and for the development of parton shower algorithms capable of incorporating multi-gluon coherence including the effects of large-angle, noncollinear emission.

Most of TMD computational tools relevant to small- $x$ and forward physics at the LHC have so far been developed within a quenched approximation in which only gluon and valence quark contributions are taken into account at TMD level $[5,6,8]$. While this gives a sensible approximation, based on the dominance of spin-1 exchange processes at high energies, to the asymptotic behavior of production processes coupled to gluons such as heavy flavor and scalar production, it is mandatory to go beyond this approximation to include preasymptotic effects, and to treat final states associated with quark-initiated processes such as Drell-Yan production. In this work we take first steps to address this issue by including sea quark contributions and examine forward Drell-Yan production.

Drell-Yan processes at the LHC are instrumental in precision electroweak measurements, in luminosity monitoring and pdf determinations, and in new physics searches [3]. Studies of Drell-Yan at TMD level have recently been performed in the framework of the soft-collinear effective theory [9-11]. Results on high-energy logarithmic corrections to Drell-Yan have been obtained in the $q g^{*}$ channel $[12,13]$ and in the associated production channel $Z / W+$ heavy quarks [14]. On the other hand, early attempts [15-18] to treat the unintegrated pdf evolution beyond the quenched approximation include quarks via splitting probabilities to lowest order of perturbation theory, neglecting any transverse momentum dependence in the branching. In [19] $\mathrm{k}_{\perp}$-dependent kinematic corrections are included, while the splitting kernels are still taken in lowest order. Also, a program to perform shower Monte Carlo evolution at unintegrated level has recently been proposed [20] based on the expansion [21] in two-particle irreducible (2PI) kernels. This program is formulated at the next-to-leading logarithmic order. However, this does not include small- $x$ logarithmic effects which are present beyond NLO in flavor singlet distributions.

The approach of the present paper is based on the high-energy form of the (off-shell) TMD quark Green function introduced in [22]. This is obtained by generalizing to finite transverse momenta, in the high-energy region, the 2PI expansion [21]. The main point is to construct unintegrated sea-quark distributions incorporating the effects of the TMD gluon-to-quark splitting kernel [22], which contains all single-logarithmic small- $x$ corrections to sea quark evolution for any order of perturbation theory. In order to relate this parton splitting kernel to forward vector boson production, we analyze the flavor exchange process at high energy according to the "reggeized quark" calculus [23,24]. This extends the effective action formalism [25], currently explored at NLO [26], to amplitudes with quark exchange in terms of effective degrees of freedom, the so-called reggeized quarks [27,28]. The use of the effective vertices [23,24] ensures gauge invariance of the coefficients relevant to perform the high-energy factorization $[22,29]$ for vector boson production, despite the off-shell parton.

We then examine kinematic effects related to the difference between the virtuality of the exchanged parton and its transverse part which, although formally subdominant both in the collinear and in the high-energy expansions, can nevertheless be numerically non-negligible. 


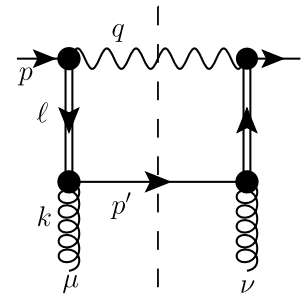

Fig. 1. The $g^{*} q \rightarrow Z q$ process within the reggeized quark formalism. Double lines with arrow indicate the effective reggeized quark exchange in the $t$-channel.

These kinematic effects are similar to those observed in the study of [19]. We perform a numerical study of the kinematic terms at the level of the partonic $Z$ cross section, and compare this with the $q g^{*} \rightarrow Z q$ result [12].

The formulation proposed in this work can be implemented in a parton shower Monte Carlo event generator. Details of the formulation and shower implementation will be described elsewhere [30]. The outline of the present paper is the following. In Section 2 we apply the reggeized quark calculus to examine $Z$-boson production and reobtain the TMD quark splitting function. In Section 3 we investigate kinematic effects in the factorization formula and study this numerically. We give conclusions in Section 4.

\section{Reggeized quark and unintegrated quark density}

In this section we relate the $Z$-boson production cross section to the unintegrated quark density defined at high energy via the transverse momentum dependent kernel [22]. For definiteness we consider factorization of the $g^{*} q \rightarrow Z q$ matrix element into off-shell point-like $q q^{*} \rightarrow Z$ coefficient and off-shell gluon-to-quark splitting function, which is of direct phenomenological relevance for forward Drell-Yan production.

Within the reggeized quark formalism of [23,24] this process can be described in the highenergy limit by the single effective diagram in Fig. 1. The double lines (with fermionic arrows) in the diagram represent the exchange of off-shell (reggeized) quarks, while the black dots represent their effective couplings to $s$-channel quarks and gauge bosons [24]. We use the following Sudakov decomposition for the initial and final momenta,

$$
\begin{aligned}
& p=x_{2} p_{2} \quad q=z x_{1} p_{1}+\left(x_{2}+\frac{t+\boldsymbol{q}^{2}}{z x_{1} s}\right) p_{2}+\boldsymbol{q}, \\
& k=x_{1} p_{1}+\boldsymbol{k} \quad p^{\prime}=(1-z) x_{1} p_{1}-\frac{t+\boldsymbol{q}^{2}}{z x_{1} s} p_{2}+\boldsymbol{k}-\boldsymbol{q},
\end{aligned}
$$

where $p_{1}$ and $p_{2}$ are light-like four-momenta respectively in the plus and minus lightcone directions, $2 p_{1} \cdot p_{2}=s$, and $\ell^{2}=t$. The reggeized quark propagators are purely transverse fermion propagators, supplemented with a projector in longitudinal momentum space,

$$
\|_{\ell=\frac{p_{2} p_{1}}{2 p_{1} \cdot p_{2}}}^{\|} \frac{i \cdot \ell}{-\ell^{2}+i \epsilon} \quad \prod_{\ell=\frac{p_{1} p_{2}}{2 p_{1} \cdot p_{2}}} \cdot \frac{i \cdot \ell}{-\ell^{2}+i \epsilon} .
$$


The couplings of the off-shell quarks to $Z$-bosons and usual quarks are

$$
\stackrel{p}{\rightarrow, \mu} \overbrace{\ell}^{q}=\frac{i e}{\cos \theta_{W} \sin \theta_{W}} \Gamma_{+}^{\mu}(p, \ell, q)\left(V_{f}-A_{f} \gamma_{5}\right),
$$

with the "plus" off-shell reggeized vertex given by

$$
\Gamma_{+}^{\mu}(p, \ell, q)=\left.\left(\gamma^{\mu}+\ell \cdot \frac{p_{1}^{\mu}}{p_{1} \cdot q}\right)\right|_{\ell \cdot p_{1}=0},
$$

where the lightcone $p_{2}$ component of the $t$-channel momentum $\ell$ is set to zero. Similarly, the lower vertex is given by

$$
\Downarrow_{\substack{\ell, \mu, a \\ \rightarrow}}^{\ell}=i g t^{a} \Gamma_{-}^{\mu}\left(k, \ell, p^{\prime}\right)
$$

with

$$
\Gamma_{-}^{\mu}\left(k, \ell, p^{\prime}\right)=\left.\left(\gamma^{\mu}+\ell \cdot \frac{p_{2}^{\mu}}{p_{2} \cdot k}\right)\right|_{\ell \cdot p_{2}=0},
$$

where the lightcone $p_{1}$ component of the $t$-channel momentum is set to zero.

The kinematic conditions $\ell \cdot p_{1}=0$ and $\ell \cdot p_{2}=0$ in Eqs. (4) and (6) reflect the strong ordering in the minus- and plus-lightcone momenta $[24,25]$ and are natural from the point of view of highenergy factorization. Using these conditions, the expression which results from Fig. 1 agrees precisely with the high-energy expansion of the $g^{*} q \rightarrow Z q$ matrix element, as carried out in [12] along the lines of the high-energy resummation [22]. However, the expression resulting from the strong ordering kinematics in Eqs. (4) and (6) only yields a good approximation in the limit of asymptotically large partonic center-of-mass energy. In the present analysis, we concentrate on a different aspect. On the one hand, we are interested in improving the description for centerof-mass energies which are not asymptotically large. On the other hand, we are interested in obtaining an off-shell (i.e., $k_{T}$-dependent) factorization in terms of the transverse-momentum dependent sea-quark distribution defined in [22] from the two-particle irreducible expansion.

To this end, we will relax some of these kinematic approximations. In the rest of this section we match the high-energy factorized expression to collinear factorization by relaxing the condition $\ell \cdot p_{2}=0$. In Section 3 we discuss the effect of relaxing the condition $\ell \cdot p_{1}=0$. Instead of Eq. (6) we consider the effective vertex with exact kinematics

$$
\tilde{\Gamma}_{-}^{\mu}\left(k, \ell, p^{\prime}\right)=i g t^{a}\left(\gamma^{\mu}+\frac{p_{2}^{\mu}}{p_{2} \cdot k} \ell\right) \quad \text { with } \ell=-z x_{1} p_{1}-\frac{\hat{t}+\boldsymbol{q}^{2}}{z x_{1} s} p_{2}-\boldsymbol{q} .
$$

Current conservation is satisfied [24,25] also in this more general case,

$$
k_{\mu} \tilde{\Gamma}_{-}^{\mu}\left(k, \ell, p^{\prime}\right) u\left(p^{\prime}\right)=i g t^{a} \not p^{\prime} u\left(p^{\prime}\right)=0 .
$$

In order to combine the above vertices with gluonic $k_{T}$ factorization [29], we contract the above vertex with the transverse gluon momentum,

$$
\tilde{\Gamma}_{-}^{\mu}\left(k, \ell, p^{\prime}\right) \cdot \frac{\boldsymbol{k}_{\mu}}{\sqrt{\boldsymbol{k}^{2}}} .
$$


This yields the $q^{*} q g^{*}$-vertex which will be used in the following. Owing to Eq. (8), this is merely a rewriting from the longitudinal polarization naturally associated with reggeized gluons to the transverse polarization, which allows one to perform the matching with the collinear factorization [21,22]. Removing the condition $\ell \cdot p_{2}=0$ for the effective vertex, we also promote the propagators in Eq. (2) to full four-dimensional propagators as follows,

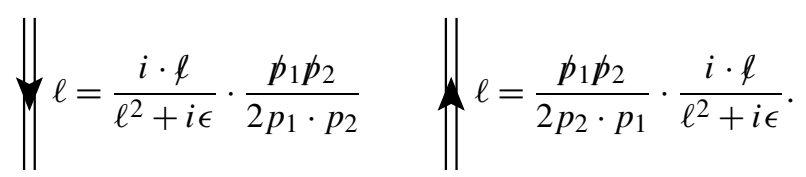

As in $[21,22]$, to perform the matching in the collinear region we include an upper bound on four-momentum exchanged in the $t$-channel, $\ell^{2}<\mu_{F}^{2}$, with $\mu_{F}$ the factorization scale.

Using the setup described above, we find that the $k_{T}$ factorized $q g^{*} \rightarrow Z q$ cross-section can be written as

$$
\sigma_{q g^{*} \rightarrow Z q}^{k_{T} \text {-fact. }}=\int_{0}^{1} d z \int \frac{d^{2} \boldsymbol{q}}{\pi} \Theta\left(\mu_{F}^{2}-\ell^{2}\right) \hat{\sigma}\left(x_{1} x_{2} s, M_{Z}^{2}, z, \boldsymbol{q}^{2}\right) R_{g^{*} q^{*}}(z, \boldsymbol{q}, \boldsymbol{k}),
$$

where $\hat{\sigma}$ is the following off-shell continuation of the pointlike $q \bar{q} \rightarrow Z$ matrix element,

$$
\hat{\sigma}\left(x_{1} x_{2} s, M_{Z}^{2}, z, \boldsymbol{q}^{2}\right)=\sqrt{2} G_{F} M_{Z}^{2}\left(V_{q}^{2}+A_{q}^{2}\right) \frac{\pi}{N_{c}} \delta\left(z x_{1} x_{2} s-\boldsymbol{q}^{2}-M_{Z}^{2}\right),
$$

with $M_{Z}$ the mass of the $Z$-boson, $G_{F}$ the Fermi coupling, $V_{q}$ and $A_{q}$ the coefficients of the vector and axial couplings of the $Z$ to a quark, and $R_{g^{*} q^{*}}(z, \boldsymbol{q}, \boldsymbol{k})$ is given by

$$
\begin{aligned}
R_{g^{*} q^{*}}(z, \boldsymbol{q}, \boldsymbol{k})= & \frac{T_{R} \alpha_{s}}{2 \pi} \frac{z(1-z)}{\left(\boldsymbol{\Delta}^{2}+z(1-z) \boldsymbol{k}^{2}\right)^{2}} \\
& \times\left[\frac{\boldsymbol{\Delta}^{2}}{z(1-z)}+4(1-2 z) \boldsymbol{\Delta} \cdot \boldsymbol{k}-4 \frac{(\boldsymbol{\Delta} \cdot \boldsymbol{k})^{2}}{\boldsymbol{k}^{2}}+4 z(1-z) \boldsymbol{k}^{2}\right],
\end{aligned}
$$

where $T_{R}=1 / 2, \boldsymbol{\Delta}=\boldsymbol{q}-z \boldsymbol{k}$. Upon azimuthal average, Eq. (13) returns the TMD gluon-toquark splitting kernel [22]. We examine azimuthal effects numerically in the next section. By replacing $R_{g^{*}} q^{*}(z, \boldsymbol{q}, \boldsymbol{k})$ with its azimuthal average and neglecting the order- $z \boldsymbol{k}$ contribution to the transverse momentum recoil in Eq. (12), we get

$$
\begin{aligned}
\sigma_{q g^{*} \rightarrow Z q}^{k_{T} \text {-fact. }} \simeq & \int_{0}^{1} d z \int \frac{d^{2} \boldsymbol{\Delta}}{\pi \boldsymbol{\Delta}^{2}} \hat{\sigma}\left(x_{1} x_{2} s, M_{Z}^{2}, z, \boldsymbol{\Delta}^{2}\right) \Theta \\
& \times\left(\mu_{F}^{2}-z \boldsymbol{k}^{2}-\frac{\boldsymbol{\Delta}^{2}}{1-z}\right) \frac{\alpha_{s}}{2 \pi} P_{q g}\left(z, \boldsymbol{k}^{2}, \boldsymbol{\Delta}^{2}\right),
\end{aligned}
$$

where

$$
\begin{aligned}
P_{q g}\left(z, \boldsymbol{k}^{2}, \boldsymbol{\Delta}^{2}\right)= & T_{R}\left(\frac{\boldsymbol{\Delta}^{2}}{\boldsymbol{\Delta}^{2}+z(1-z) \boldsymbol{k}^{2}}\right)^{2} \\
& \times\left[(1-z)^{2}+z^{2}+4 z^{2}(1-z)^{2} \frac{\boldsymbol{k}^{2}}{\boldsymbol{\Delta}^{2}}\right] .
\end{aligned}
$$


It was pointed out in [22] that Eq. (15) allows one to relate the sea quark distribution, with nextto-leading logarithmic accuracy at small $x$, to the small- $x$ gluon Green's function $\mathcal{G}\left(x, \boldsymbol{k}^{2}, \mu^{2}\right)$ obeying the BFKL equation. Although evaluated off-shell, the splitting function in Eq. (15) is universal [22,31]. It reduces to the collinear splitting function at lowest order for $\boldsymbol{k}=0$, but in the high-energy limit it factorizes correctly the finite $\boldsymbol{k}$ dependence to all orders. By using this result, the unintegrated sea quark distribution is given by

$$
\begin{aligned}
\mathcal{Q}^{\text {sea }}\left(x, \frac{\boldsymbol{\Delta}^{2}}{\mu^{2}}, \frac{\mu_{F}^{2}}{\mu^{2}}\right)= & \int_{x}^{1} \frac{d z}{z} \int d \boldsymbol{k}^{2} \Theta\left(\mu_{F}^{2}-\frac{\boldsymbol{\Delta}^{2}+z(1-z) \boldsymbol{k}^{2}}{1-z}\right) \\
& \times \frac{1}{\boldsymbol{\Delta}^{2}} \frac{\alpha_{s}}{2 \pi} P_{q g}\left(z, \boldsymbol{k}^{2}, \boldsymbol{\Delta}^{2}\right) \mathcal{G}\left(\frac{x}{z}, \boldsymbol{k}^{2}, \mu^{2}\right) .
\end{aligned}
$$

We thus write the forward $Z$-boson hadronic cross section in the high energy factorized form

$$
\sigma_{p p \rightarrow Z}\left(s, M_{Z}^{2}\right)=\sum_{j} \int_{0}^{1} d x_{1} \int \frac{d^{2} \boldsymbol{\Delta}}{\pi} \int_{0}^{1} d x_{2} \hat{\sigma} \mathcal{Q}^{\mathrm{sea}}\left(x_{1}, \frac{\boldsymbol{\Delta}^{2}}{\mu^{2}}, \frac{\mu_{F}^{2}}{\mu^{2}}\right) Q^{(j)}\left(x_{2}, \frac{\mu_{F}^{2}}{\mu^{2}}\right),
$$

with $\mathcal{Q}^{\text {sea }}$ given in Eq. (16) and $Q^{(j)}$ the (integrated) parton distribution as provided by collinear factorization. The scale $\mu^{2}$ in Eqs. (16) and (17) is an initial infra-red scale at which the parton distribution functions are defined. The dependence on this scale occurs for the TMD distributions as a direct consequence of the matching procedure between high energy and collinear factorization. The presence of collinear singularities in the integrations over momentum lines connecting the 2PI amplitudes requires the use of a regulator for the BFKL gluon Green's function which breaks the scale invariance of the LO BFKL equation. In addition to the BFKL gluon density, also the integral over $\boldsymbol{\Delta}^{2}$ in Eq. (17) is collinear divergent in the limit $\boldsymbol{k}^{2} \rightarrow 0$ and requires regularization, leading to an additional dependence on the scale $\mu^{2}$. While in [22] this is achieved through dimensional regularization in $d=4+2 \epsilon$ dimensions, a cut-off regularization will be used in future Monte Carlo realizations of Eq. (16). The dependence on the scale $\mu$ cancels up to second order corrections, i.e. to order $\alpha_{s}\left(\alpha_{s} \ln 1 / x\right)^{n}$ which is the accuracy at which Eqs. (16) and (17) are valid.

We will be interested in applications of the sea quark distribution in Eq. (16) to shower Monte Carlo generators including the transverse momentum dependence of the parton branching, such as [32]. Compared to previous TMD approaches [15,17,20], this distribution includes the finite- $\boldsymbol{k}$ dependence of the kernel (15), responsible for all-order logarithmically enhanced corrections to quark evolution at small $x$. For these applications it is relevant to investigate the size of corrections associated with the high-energy kinematic approximations discussed earlier. We turn to this in the next section, and study the numerical effect of the kinematic contributions on the off-shell $Z$-boson production cross section.

\section{Kinematic effects in the partonic $Z$ cross section}

In this section we discuss that the accuracy of the factorized expression obtained above can be increased by going beyond the strong ordering approximation in Eqs. (3), (4). We will write the convolution integral in terms of the virtuality of the four-momentum exchanged in the $t$-channel, $|t|=-\ell^{2}$, rather than the transverse momentum. While the two formulations coincide in 
the asymptotic high-energy limit $z \rightarrow 0$, they differ by inclusion of finite- $z$ terms. We examine numerically the role of these terms.

The formulation in $|t|$ arises naturally if we remove the constraint $\ell \cdot p_{1}=0$ in the effective vertex of Eq. (4). The kinematically improved version of this vertex is

$$
\tilde{\Gamma}_{+}^{\mu}(p, \ell, q)=\left(\gamma^{\mu}+\ell \cdot \frac{p_{1}^{\mu}}{p_{1} \cdot q}\right) .
$$

Gauge invariance can be verified similarly to Eqs. (8), (9). One then recovers Eq. (11) with $\hat{\sigma}$ replaced by

$$
\hat{\sigma}^{t}=\sqrt{2} G_{F} M_{Z}^{2}\left(V_{q}^{2}+A_{q}^{2}\right) \frac{\pi}{N_{c}} \delta\left(z x_{1} x_{2} s+t-M_{Z}^{2}\right)
$$

where

$$
-t=-\ell^{2}=\frac{\Delta^{2}}{1-z}+z \boldsymbol{k}^{2}
$$

The angular average can now be performed exactly, and yields

$$
\sigma_{q g^{*} \rightarrow Z q}^{t}=\int_{0}^{1} d z \int_{0}^{\infty} \frac{d \boldsymbol{\Delta}^{2}}{\boldsymbol{\Delta}^{2}} \hat{\sigma}^{t} \Theta\left(\mu_{F}^{2}-\frac{\boldsymbol{\Delta}^{2}+z(1-z) \boldsymbol{k}^{2}}{1-z}\right) \frac{\alpha_{s}}{2 \pi} P_{q g}\left(z, \boldsymbol{k}^{2}, \boldsymbol{\Delta}^{2}\right) .
$$

Unlike Eq. (14) the above expression no longer decouples in transverse momentum space. However, by changing variable to $|t|$ in the convolution integral, we get

$$
\begin{aligned}
\sigma_{q g^{*} \rightarrow Z q}^{t}\left(x_{1} x_{2} s, \boldsymbol{k}^{2}, M_{Z}^{2}\right)= & \int_{0}^{1} d z \int \frac{d|t|}{|t|-z \boldsymbol{k}^{2}} \Theta\left(\mu_{F}^{2}-|t|\right) \hat{\sigma}\left(x_{1} x_{2} s, M_{Z}^{2}, z,|t|\right) \\
& \times \Theta\left(|t|-z \boldsymbol{k}^{2}\right) \frac{\alpha_{s}}{2 \pi} P_{q g}\left(z, \boldsymbol{k}^{2},(1-z)\left(|t|-z \boldsymbol{k}^{2}\right)\right) .
\end{aligned}
$$

This result shares some features with the results in [17] and [19]. In particular, as in [19] it takes into account subleading corrections to strong ordering by keeping track of the exact kinematic relation between virtuality and transverse momentum. It was shown in the numerical NLO-DGLAP analysis of [19] that these kinematic contributions provide a large fraction of the full corrections at NLO. On the other hand, unlike [17,19] Eq. (22) contains the dynamical effects of transverse momentum dependent terms in the gluon-to-quark splitting kernel. These become potentially important in the forward production kinematics, as they give rise to logarithmically enhanced terms for small $x$ at higher orders in $\alpha_{s}$.

While the general structure of the unintegrated sea quark density Eq. (16) remains unchanged, the off-shellness is now expressed in terms of the absolute value of the four momentum square exchanged in the $t$-channel,

$$
\begin{aligned}
\mathcal{Q}_{t}^{\text {sea }}\left(x, \frac{|t|}{\mu^{2}}, \frac{\mu_{F}^{2}}{\mu^{2}}\right)= & \int_{x}^{1} \frac{d z}{z} \int d \boldsymbol{k}^{2} \Theta\left(|t|-z \boldsymbol{k}^{2}\right) P_{q g}\left(z, \boldsymbol{k}^{2},(1-z)\left(|t|-z \boldsymbol{k}^{2}\right)\right) \\
& \times \frac{1}{|t|-z \boldsymbol{k}^{2}} \frac{\alpha_{s}}{2 \pi} \mathcal{G}\left(\frac{x}{z}, \boldsymbol{k}^{2}, \mu^{2}\right) .
\end{aligned}
$$


In [22,31] the low- $\boldsymbol{k}^{2}$ behavior of Eqs. (16), (23) is analyzed using dimensional regularization. For the parton-shower applications [30] an infrared cut-off is applied. In the numerical study that follows, we examine the effect of the TMD sea quark distribution on the $Z$-boson partonic cross section. For this purpose we will work at fixed $\boldsymbol{k}^{2}$.

We perform a numerical comparison of Eqs. (14) and (22), corresponding respectively to the kinematic effects of going beyond the plus-momentum strong ordering and the minus-momentum strong ordering in the high-energy factorized formula. We compare this with the full $q g^{*} \rightarrow q Z$ matrix element result. We consider also the result of the collinear approximation obtained by setting $\boldsymbol{k}^{2}=0$ in Eq. (14). We examine the dependence on the transverse momentum variable $\boldsymbol{\Delta}=\boldsymbol{q}-z \boldsymbol{k}$. Here $\boldsymbol{q}$ is the vector boson transverse momentum; $z$ is the plus-momentum fraction transferred to the vector boson from the incoming gluon that results from the small- $x$ initial-state shower; $\boldsymbol{k}$ is the transverse momentum carried by the partons emitted in this shower (i.e., partons radiated in addition to the leading quark against which the vector boson recoils). In Fig. 2 and Fig. 3 we examine respectively the region where $|\boldsymbol{\Delta}|$ is small compared to the $Z$-boson mass $M_{Z}$ and the region where $|\boldsymbol{\Delta}|$ is on the order of the $Z$-boson mass or larger.

For small $|\boldsymbol{\Delta}|$ we find that the differences between Eq. (14) and Eq. (22) are numerically small, and that both expressions are close to the full result; as $|\boldsymbol{\Delta}|$ increases, we find that the deviations due to the kinematic contributions by which Eq. (14) and Eq. (22) differ become non-negligible, and that Eq. (22) gives a better approximation to the full result. Fig. 2 illustrates the small $|\boldsymbol{\Delta}|$ region. In Fig. 2(a) we plot the differential cross section $d \sigma / d \boldsymbol{\Delta}^{2}$ for fixed values of $\boldsymbol{k}^{2}$ and $x_{1} x_{2} s$. The solid, dashed and dot-dashed curves overlap on the scale of this plot, while the dotted curve, corresponding to the collinear approximation, deviates from them when $|\boldsymbol{\Delta}| \lesssim|\boldsymbol{k}|$. In Fig. 2(b) we zoom in on the small relative deviations of Eq. (14) and Eq. (22) from the full result for the differential cross section $d \sigma / d \boldsymbol{\Delta}^{2}$. We see that throughout the range shown in Fig. 2(b) the deviations are at most of the order of few percent. In this region the factorized expressions based on reggeized quark graphs are close to the full result, independently of the different kinematic approximations on the longitudinal momentum orderings. What dominates this region are the transverse momentum dependent terms, which are kept correctly by both Eq. (14) and Eq. (22). On the other hand, we see in Fig. 2(a) that in the small $|\boldsymbol{\Delta}|$ region the collinear expression obtained by setting $\boldsymbol{k}^{2}=0$ in Eq. (14) is far from the full result, due to missing TMD corrections to the kernel (15).

As $|\boldsymbol{\Delta}|$ increases the kinematic effects from the plus-momentum ordering and minusmomentum ordering become significant. Fig. 3 illustrates these effects for $|\boldsymbol{\Delta}|$ on the order of the $Z$-boson mass $M_{Z}$, or larger. Note that in this region the collinear $\boldsymbol{k}^{2}=0$ expression gives a good approximation to Eq. (14). The dashed and dotted curves nearly overlap on the scale of the plots in Fig. 3. However, the full result contains significant corrections with respect to these curves, corresponding to terms that are subdominant both in the collinear and in the high-energy expansions. We see that a non-negligible part of these corrections can be taken into account by including kinematic contributions from terms beyond the strong ordering in the lightcone minusmomenta, as Eq. (22) does. This is analogous to effects observed in [19]. As a result Eq. (22) provides a closer approximation to the full matrix element. It can be verified numerically that the corrections die out in the limit of asymptotically large $s$. This analysis is however significant for applications to finite collider energies.

We thus observe that taking into account TMD terms in the parton splitting and the reggeized quark coefficient enables one to extend the description of vector boson production in terms of factorized quark distributions from the collinear region into the region where $|\boldsymbol{\Delta}|$ is of order $|\boldsymbol{k}|$ and transverse momenta are no longer strongly ordered. This is a region where collinear 


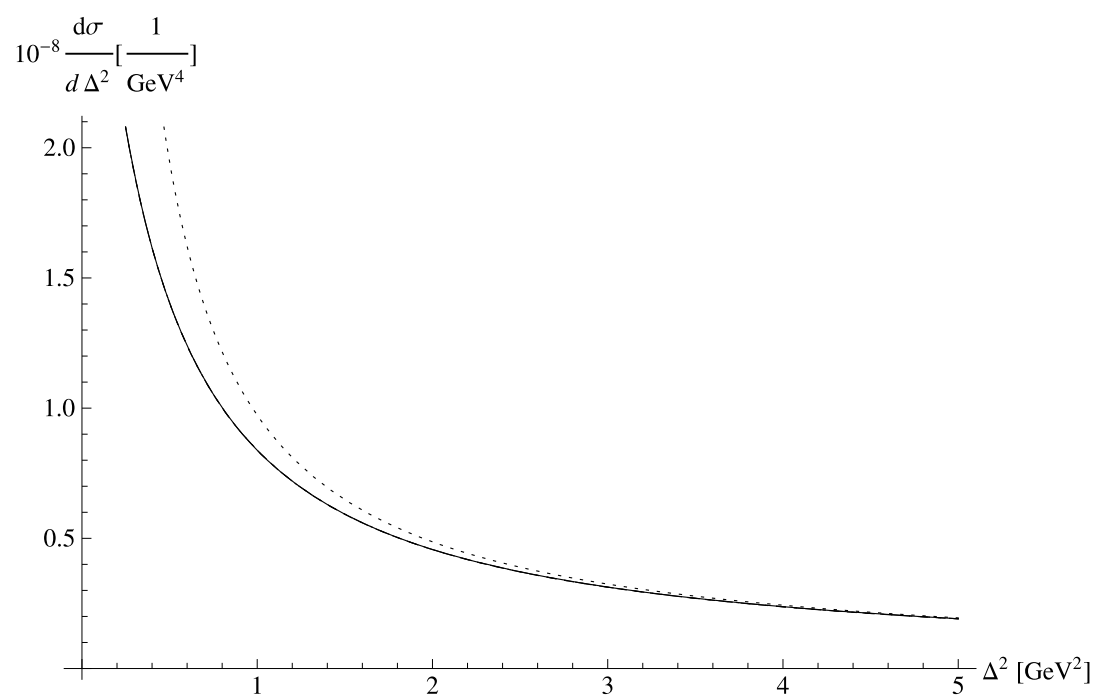

(a)

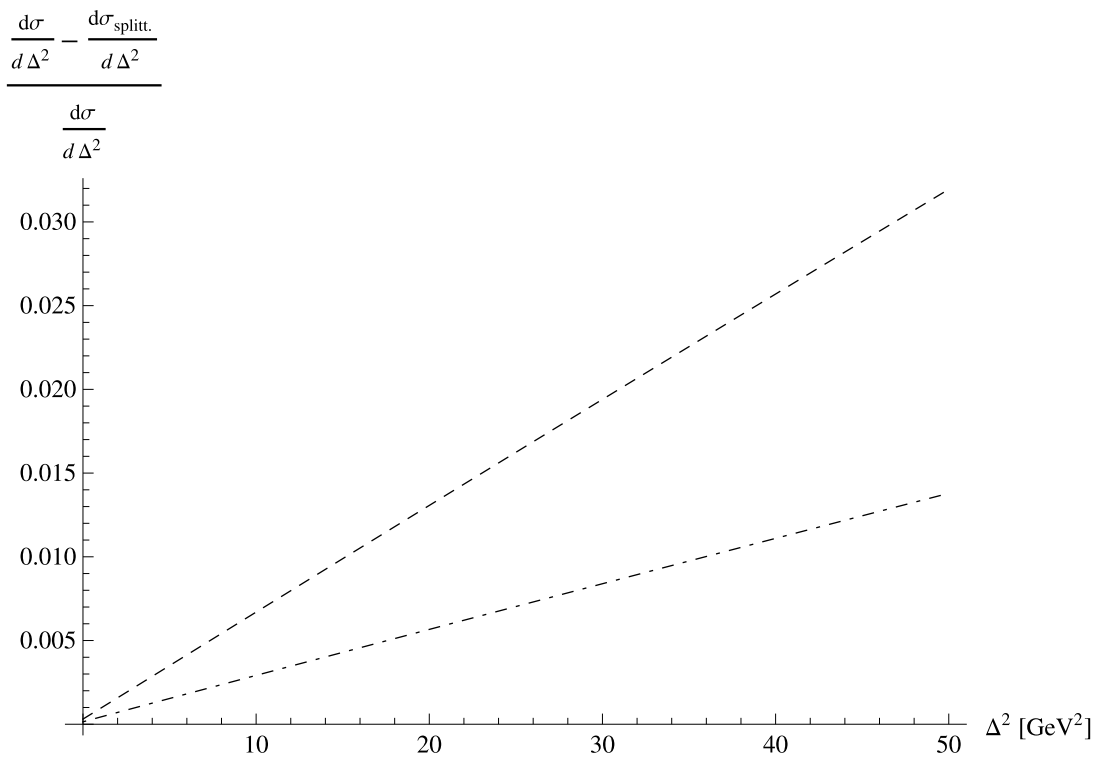

(b)

Fig. 2. (a) $\boldsymbol{\Delta}^{2}$ dependence of the differential cross section $d \sigma / d \boldsymbol{\Delta}^{2}$ for small $|\boldsymbol{\Delta}|$ : (solid) full; (dashed) no plusmomentum ordering; (dot-dashed) no plus-momentum and minus-momentum ordering; (dotted) collinear approximation. All but the last curve overlap in this region. We set $x_{1} x_{2} s=2.5 M_{Z}^{2}, k^{2}=2 \mathrm{GeV}^{2}$. (b) Relative deviations in the differential cross section $d \sigma / d \boldsymbol{\Delta}^{2}$ : (dashed) no plus-momentum ordering; (dot-dashed) no plus-momentum and minusmomentum ordering.

approximations are seen not to be sufficient, and it is relevant to forward Drell-Yan. We will use the unintegrated quark distribution thus defined in forthcoming studies [30]. As a result of TMD terms, on the other hand, the initial-state kinematics becomes rather more complex. Corrections to longitudinal momentum ordering in the effective vertices are seen to be numerically significant 


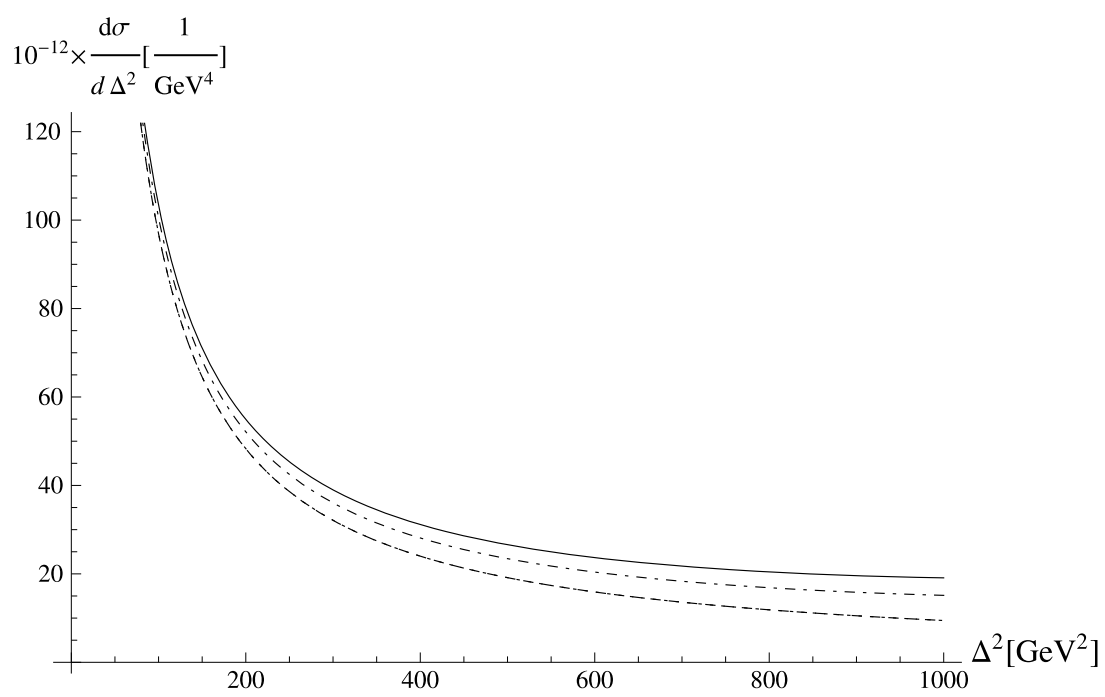

(a)

$10^{-12} \times \frac{\mathrm{d} \sigma}{d \Delta^{2}}\left[\frac{1}{\mathrm{GeV}^{4}}\right]$

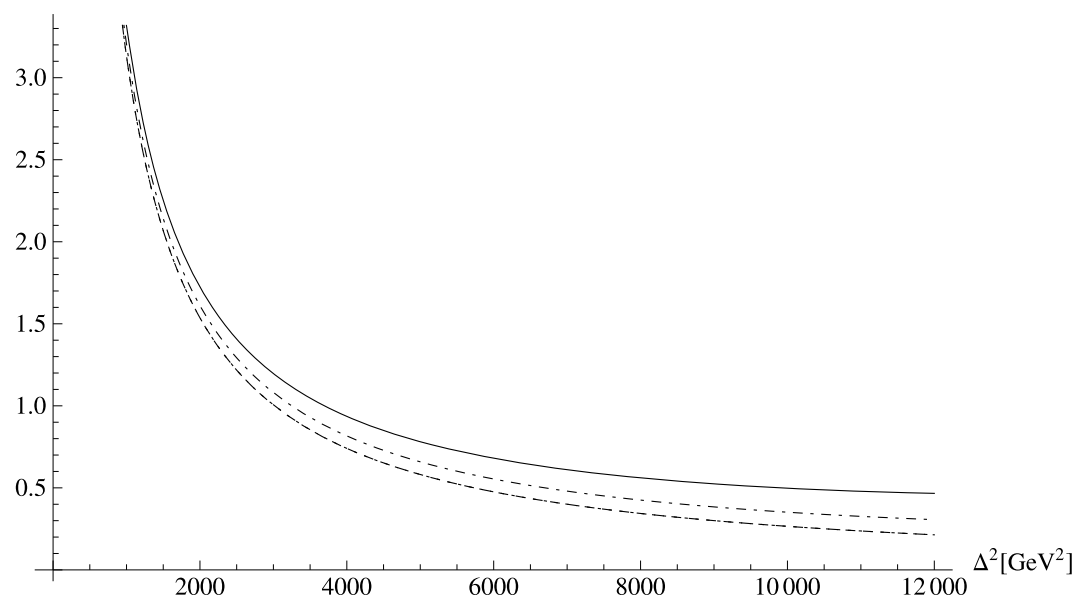

(b)

Fig. 3. $\boldsymbol{\Delta}^{2}$ dependence of the differential cross section $d \sigma / d \boldsymbol{\Delta}^{2}$ in the large $|\boldsymbol{\Delta}|$ region: (solid) full; (dashed) no plusmomentum ordering; (dot-dashed) no plus-momentum and minus-momentum ordering; (dotted) collinear approximation. We set $\boldsymbol{k}^{2}=2 \mathrm{GeV}^{2}$; (a) $x_{1} x_{2} s=2.5 M_{Z}^{2}$, (b) $x_{1} x_{2} s=12.5 M_{Z}^{2}$.

when $|\boldsymbol{\Delta}|$ is of order the vector boson mass. To this end it is useful to employ the formulation given in this section which includes the exact one-loop kinematics.

\section{Conclusion}

Drell-Yan production processes are instrumental in hadron collider experiments both for hadronic physics studies and for new physics searches. At the LHC a new kinematic region 
opens up for forward Drell-Yan, in which the QCD treatment of the production process and associated final states acquires new features due to multi-parton radiation over long rapidity intervals. The emission of soft gluons that are not collinearly ordered becomes relevant, and effects of color coherence set in associated with the region of small longitudinal momentum fractions $x$. Taking this into account calls for improved formulations of factorization in QCD at unintegrated, or transverse momentum dependent, level.

Fully general TMD factorization formulas are still lacking [1,2], due to the difficulty in disentangling systematically soft and collinear gluon correlations between initial and final states. This is illustrated, for instance, by back-to-back di-hadron and di-jet hadroproduction [33,34]. In the case of small $x$, however, TMD factorization results exist [25,29] based on dominance of single gluon helicity at high energy. These results can be used to define gauge-invariantly the TMD gluon distribution. See recent works in $[6,35,36]$. In this paper we have used the method [22] to extend the above treatment to the TMD sea-quark distribution. This is the dominant channel coupling to forward Drell-Yan production. We have related the TMD splitting kernel [22] to the forward Z-boson cross section. The reggeized quark formalism [23,24] is used to treat the offshell quark coefficient and ensure gauge invariance. We have analyzed numerically effects due to the transverse momentum kinematics in the relationship between the $Z$-boson spectrum and the TMD sea-quark distribution.

Phenomenological applications of the reggeized quark formalism were investigated in [28] using the approach $[16,17]$ to TMD pdfs. Compared to these studies, the main feature of our approach is that it includes, besides the lowest-order quark splitting function, the full series of finite- $k_{\perp}$ terms in the TMD kernel. These terms control the perturbative summation of small- $x$ logarithmic corrections to flavor-singlet observables to all orders in $\alpha_{s}$.

Future extensions of the results above involve several directions. One concerns large- $x$ corrections, likely to be important for Drell-Yan phenomenology. See [11,37-39] for discussion of $x \rightarrow 1$ issues in TMD quark distributions. Another direction concerns nonlinear effects from high parton densities in the small- $x$ region. Recent works $[40,41]$ study multiple scattering contributions in dense targets and nuclei. Techniques such as [42] have been proposed to incorporate the treatment of multiple-gluon rescattering graphs at small- $x$ starting from the operator matrix elements [1,2] for parton distributions. They may be helpful for extensions to the high-density region that are aimed at retaining accuracy also in the treatment of contributions from high $\mathrm{p}_{\mathrm{T}}$ processes, e.g. Drell-Yan on nuclei [43].

The results in this paper can be implemented in a parton shower Monte Carlo generator including transverse-momentum dependent branching, such as [32]. Work along these lines is in progress [30]. This will allow one to address predictions for exclusive final-state observables associated with forward Drell-Yan production, for which intense experimental activity is forthcoming [44].

\section{Acknowledgements}

M.H. is grateful for financial support from the German Academic Exchange Service (DAAD), the MICINN under grant FPA2010-17747, the Research Executive Agency (REA) of the European Union under the Grant Agreement number PITN-GA-2010-264564 (LHCPhenoNet) and the Helmholtz Terascale Analysis Center. F.H. thanks the CERN Theory Division for hospitality and support. 


\section{References}

[1] J.C. Collins, Foundations of Perturbative QCD, CUP, 2011.

[2] S. Mert Aybat, T.C. Rogers, Phys. Rev. D 83 (2011) 114042.

[3] Z. Ajaltouni, et al., Proceedings of the Workshop HERA and the LHC, CERN/DESY, arXiv:0903.3861 [hep-ph], 2008.

[4] M. Deak, et al., JHEP 0909 (2009) 121, arXiv:0908.1870 [hep-ph];

F. Hautmann, arXiv:0909.1250 [hep-ph];

F. Hautmann, PoS ICHEP 2010 (2010) 108, arXiv:1101.2656 [hep-ph].

[5] M. Deak, et al., arXiv:1012.6037 [hep-ph];

M. Deak, et al., arXiv:1112.6354 [hep-ph];

M. Deak, et al., arXiv:1112.6386 [hep-ph].

[6] E. Avsar, arXiv:1108.1181 [hep-ph];

E. Avsar, arXiv:1203.1916 [hep-ph].

[7] F. Hautmann, H. Jung, Nucl. Phys. B (Proc. Suppl.) 184 (2008) 64, arXiv:0808.0873 [hep-ph];

F. Hautmann, Acta Phys. Polon. B 40 (2009) 2139;

F. Hautmann, PoS ICHEP 2010 (2010) 150.

[8] F. Hautmann, H. Jung, JHEP 0810 (2008) 113, arXiv:0804.1746 [hep-ph].

[9] T. Becher, M. Neubert, Eur. Phys. J. C 71 (2011) 1665.

[10] S. Mantry, F. Petriello, Phys. Rev. D 83 (2011) 053007;

S. Mantry, F. Petriello, Phys. Rev. D 84 (2011) 014030, arXiv:1108.3609 [hep-ph];

Y. Li, S. Mantry, F. Petriello, Phys. Rev. D 84 (2011) 094014.

[11] M. Garcia-Echevarria, A. Idilbi, I. Scimemi, Phys. Rev. D 84 (2011) 011502, arXiv:1111.4996 [hep-ph]; A. Idilbi, I. Scimemi, Phys. Lett. B 695 (2011) 463, arXiv:1012.4419 [hep-ph].

[12] R.D. Ball, S. Marzani, Nucl. Phys. B 814 (2009) 246, arXiv:0906.4729 [hep-ph].

[13] A.V. Lipatov, M.A. Malyshev, N.P. Zotov, JHEP 1112 (2011) 117.

[14] M. Deak, F. Schwennsen, JHEP 0809 (2008) 035; S.P. Baranov, A.V. Lipatov, N.P. Zotov, Phys. Rev. D 78 (2008) 014025.

[15] A. Gawron, J. Kwiecinski, W. Broniowski, Phys. Rev. D 68 (2003) 054001.

[16] M.A. Kimber, A.D. Martin, M.G. Ryskin, Phys. Rev. D 63 (2001) 114027; M.A. Kimber, A.D. Martin, M.G. Ryskin, Eur. Phys. J. C 12 (2000) 655.

[17] A.D. Martin, M.G. Ryskin, G. Watt, Phys. Rev. D 70 (2004) 014012; A.D. Martin, M.G. Ryskin, G. Watt, Eur. Phys. J. C 31 (2003) 73.

[18] S. Höche, F. Krauss, T. Teubner, Eur. Phys. J. C 58 (2008) 17.

[19] A.D. Martin, M.G. Ryskin, G. Watt, Eur. Phys. J. C 66 (2010) 163.

[20] S. Jadach, et al., arXiv:1004.4131 [hep-ph]; S. Jadach, et al., arXiv:1002.0010 [hep-ph];

S. Jadach, M. Skrzypek, arXiv:0909.5588 [hep-ph];

S. Jadach, M. Skrzypek, arXiv:0905.1399 [hep-ph].

[21] G. Curci, W. Furmanski, R. Petronzio, Nucl. Phys. B 175 (1980) 27.

[22] S. Catani, F. Hautmann, Nucl. Phys. B 427 (1994) 475;

S. Catani, F. Hautmann, Phys. Lett. B 315 (1993) 157.

[23] L.N. Lipatov, M.I. Vyazovsky, Nucl. Phys. B 597 (2001) 399.

[24] A.V. Bogdan, V.S. Fadin, Nucl. Phys. B 740 (2006) 36.

[25] L.N. Lipatov, Nucl. Phys. B 452 (1995) 369; L.N. Lipatov, Phys. Rep. 286 (1997) 131.

[26] M. Hentschinski, A. Sabio Vera, Phys. Rev. D 85 (2012) 056006, arXiv:1110.6741 [hep-ph]; M. Hentschinski, Nucl. Phys. B 859 (2012) 129, arXiv:1112.4509 [hep-ph];

G. Chachamis, M. Hentschinski, J.D. Madrigal Martinez, A. Sabio Vera, Nucl. Phys. B 861 (2012) 133, arXiv: 1202.0649 [hep-ph].

[27] V.S. Fadin, V.E. Sherman, Zh. Eksp. Teor. Fiz. 72 (1977) 1640;

V.S. Fadin, V.E. Sherman, Pisma Zh. Eksp. Teor. Fiz. 23 (1976) 599.

[28] B.A. Kniehl, V.A. Saleev, A.V. Shipilova, E.V. Yatsenko, Phys. Rev. D 84 (2011) 074017; B.A. Kniehl, V.A. Saleev, A.V. Shipilova, Phys. Rev. D 79 (2009) 034007; V.A. Saleev, Phys. Rev. D 80 (2009) 114016; V.A. Saleev, Phys. Rev. D 78 (2008) 114031. 
[29] S. Catani, M. Ciafaloni, F. Hautmann, Phys. Lett. B 307 (1993) 147;

S. Catani, M. Ciafaloni, F. Hautmann, Nucl. Phys. B 366 (1991) 135;

S. Catani, M. Ciafaloni, F. Hautmann, Phys. Lett. B 242 (1990) 97.

[30] F. Hautmann, M. Hentschinski, H. Jung, in preparation.

[31] M. Ciafaloni, D. Colferai, JHEP 0509 (2005) 069.

[32] H. Jung, et al., Eur. Phys. J. C 70 (2010) 1237.

[33] P.J. Mulders, T.C. Rogers, Phys. Rev. D 81 (2010) 094006.

[34] W. Vogelsang, F. Yuan, Phys. Rev. D 76 (2007) 094013;

J.C. Collins, arXiv:0708.4410 [hep-ph];

A. Bacchetta, C.J. Bomhof, P.J. Mulders, F. Pijlman, Phys. Rev. D 72 (2005) 034030.

[35] F. Dominguez, A.H. Mueller, S. Munier, B.W. Xiao, Phys. Lett. B 705 (2011) 106.

[36] F. Dominguez, J.W. Qiu, B.W. Xiao, F. Yuan, Phys. Rev. D 85 (2012) 045003.

[37] F.A. Ceccopieri, Mod. Phys. Lett. A 24 (2009) 3025, arXiv:1006.4731 [hep-ph];

F.A. Ceccopieri, L. Trentadue, Phys. Lett. B 660 (2008) 43;

F.A. Ceccopieri, L. Trentadue, Phys. Lett. B 636 (2006) 310.

[38] I.O. Cherednikov, N.G. Stefanis, Nucl. Phys. B 802 (2008) 146;

I.O. Cherednikov, N.G. Stefanis, Phys. Rev. D 77 (2008) 094001;

I.O. Cherednikov, N.G. Stefanis, Phys. Rev. D 80 (2009) 054008;

I.O. Cherednikov, N.G. Stefanis, arXiv:0911.1031 [hep-ph];

I.O. Cherednikov, A.I. Karanikas, N.G. Stefanis, Nucl. Phys. B 840 (2010) 379.

[39] J.C. Collins, F. Hautmann, JHEP 0103 (2001) 016;

J.C. Collins, F. Hautmann, Phys. Lett. B 472 (2000) 129;

F. Hautmann, Nucl. Phys. B 604 (2001) 391;

F. Hautmann, Phys. Lett. B 655 (2007) 26, arXiv:0708.1319 [hep-ph].

[40] F. Dominguez, C. Marquet, B.W. Xiao, F. Yuan, Phys. Rev. D 83 (2011) 105005.

[41] A. Stasto, B.W. Xiao, D. Zaslavsky, arXiv:1204.4861 [hep-ph].

[42] F. Hautmann, D.E. Soper, Phys. Rev. D 75 (2007) 074020;

F. Hautmann, D.E. Soper, arXiv:0712.0526 [hep-ph];

F. Hautmann, D.E. Soper, Phys. Rev. D 63 (2000) 011501;

F. Hautmann, Phys. Lett. B 643 (2006) 171;

F. Hautmann, arXiv:0812.2873 [hep-ph];

F. Hautmann, arXiv:hep-ph/0209320;

F. Hautmann, arXiv:hep-ph/0105082;

F. Hautmann, et al., arXiv:hep-ph/9906284;

F. Hautmann, et al., arXiv:hep-ph/9806298.

[43] H. Paukkunen, C.A. Salgado, JHEP 1103 (2011) 071.

[44] LHCb Collaboration, preprint LHCb-CONF-2012-013;

LHCb Collaboration, preprint LHCb-CONF-2011-039;

CMS Collaboration, Phys. Rev. D 85 (2012) 032002;

ATLAS Collaboration, Phys. Rev. D 85 (2012) 072004. 